Document downloaded from:

http://hdl.handle.net/10251/98783

This paper must be cited as:

Domingo Beltran, M.; Luna Molina, R.; Satorre, MÁ.; Santonja Moltó, MDC.; Millán Verdú, C. (2015). Experimental Measurement of Carbon Dioxide Polarizability in the Solid State. Journal of Low Temperature Physics. 181(1):1-9. doi:10.1007/s10909-015-1326-6

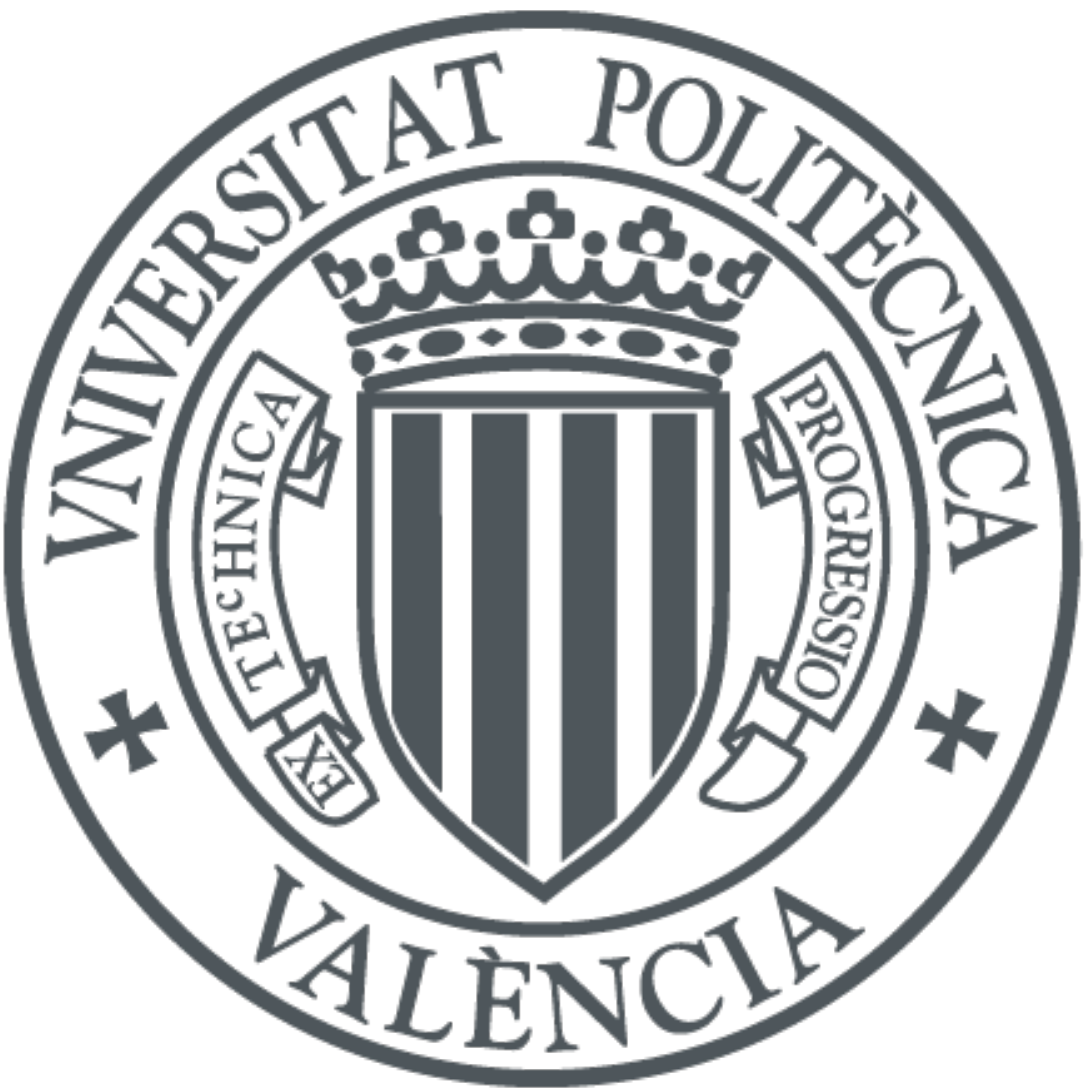

The final publication is available at

http://doi.org/10.1007/s10909-015-1326-6

Copyright Springer-Verlag

Additional Information 


\title{
Experimental measurement of carbon dioxide polarizability in the solid state
}

\author{
M. Domingo, ${ }^{\text {a) }}$ R. Luna, M.A. Satorre, C. Santonja, and C. Millán \\ Centro de Tecnologías Físicas, Universitat Politècnica de València, 46022 Valencia, Spain
}

\begin{abstract}
We have experimentally determined the polarizability of $\mathrm{CO}_{2}$ using the Lorentz-Lorenz equation by simultaneously measuring the density and the refractive index. The $\mathrm{CO}_{2}$ conditions were solid phase, $10^{-7}$ mbar pressure, and temperature range 10-86 K. The polarizability value compares well with previous gas-phase experimental results and the results from simulations, and does not depend on the temperature of $\mathrm{CO}_{2}$ ice formation. This value is constant in the temperature range studied, despite a structural change from amorphous to crystalline.
\end{abstract}

Keywords: Polarizability, experimental, Lorentz-Lorenz, quartz crystal microbalance, density, refractive index.

\section{INTRODUCTION}

There are multiple electrical properties that can be studied to determine the electronic structure of an atom or molecule. Some of the most basic are the dipole and quadrupole moments, and the polarizability, which is the focus of this article. The dipole moment gives a measure of the asymmetry of the distribution of molecular charge, and it is expressed as a vector. The quadrupole moment is a quantity that measures the shape of the electron distribution, and it is expressed by a second-order tensor.

The polarizability of an atom or molecule is the physical magnitude that describes the response of the electron cloud to an external field. The energy shift $\Delta W$ of the atom or molecule caused by an external electric field $E$ is proportional to $E^{2}$ when $E$ is weak compared with the internal electric field between the nucleus and electron cloud. The constant of proportionality is the electric dipole polarizability $\alpha$, which is defined by $\Delta W=-\alpha E^{2} / 2$, where the induced electric dipole is $\alpha E$. Hyperpolarizabilities, i.e., coefficients of higher powers of $E$, are less often required. The polarizability magnitude is a second-order tensor [1] that in spherically symmetric charge distributions reduces to a single number. In any case, an average polarizability is usually adequate in calculations. These magnitudes are the subject of theoretical and experimental study.

The polarizability can be experimentally measured with different techniques. These all have the common feature of measurement of the refractive index and the density, which, when used in the Lorentz-Lorenz equation, gives the polarizability. For example,

a) Author to whom correspondence should be addressed. Electronic mail: mdomingo@fis.upv.es 
the refraction can be measured using a Fabry-Perot refractometer [2], by the change in the resonant frequency of a chamber filled with the substance under study [3], or by the difference in the capacitance of a capacitor that is empty or filled with the substance [4]. The density is not always measured under the same conditions as the refractive index, but its value is usually extrapolated by an equation of state.

In this study, we performed gas deposition experiments under conditions of low temperature (10 K) and high vacuum (10 $\left.{ }^{-7} \mathrm{mbar}\right)$ to produce $\mathrm{CO}_{2}$ ice. We simultaneously measured the density and the refractive index of the ice under the same conditions using a quartz crystal microbalance (QCMB) and two $632.8 \mathrm{~nm}$ He-Ne lasers using the double-laser interference technique [5]. These values and the Lorentz-Lorenz equation enabled us to obtain the polarizability.

The theoretical study of the charge distribution and polarizability can be carried out using electromagnetic theory [6] or through $a b$ initio quantum mechanics calculations [7][8]. To determine the electron density distribution, Hartree-Fock theory, Moller-Plesset perturbation theory, and quadratic configuration interaction theory [9] can be used. However, the results of these simulations need to be validated by comparing with experimental results. It is also important to have accurate values for the polarizability because they can be used to obtain other values, such as molecular anisotropy [10], the Kerr effect constant [11], and even the quadrupole moment [12].

Here, we focus on carbon dioxide $\left(\mathrm{CO}_{2}\right)$ because it is important for reactions, such as hydrolysis [13], in terrestrial [14] and astrophysical [15] scenarios, and because of its applications [16]. $\mathrm{CO}_{2}$ is a symmetrical and linear molecule, and it is therefore non-polar and has no permanent dipole moment. This makes it especially suitable for theoretical study.

\section{EXPERIMENTAL SETUP}

A diagram of the experimental apparatus [5] [17] showing the high-vacuum chamber that contains the cryocooled substrate, the vacuum pre-chamber for preparing the molecule under study, and the position of the lasers and photodiodes is shown in Fig. 1. A QCMB covered with a planar gold film that was optically thick for the He-Ne laser wavelength was used. The QCMB was put in thermal contact with a closed-cycle helium refrigerator and was placed inside a $10 \mathrm{~cm}$ deep high vacuum chamber that routinely operates in the $10^{-8}-10^{-7}$ mbar range. This pressure was obtained by continuous evacuation with a turbomolecular pump backed with a rotary mechanical pump.

The cooling system used was a double-stage cold head that cooled the QCMB to $10 \mathrm{~K}$. A resistor was connected to the end of the second stage. With the collaborative work of the cryostat (always trying to cool to $10 \mathrm{~K}$ ) and the heat from the resistor with a controlled intensity, the temperature could be varied from 10 to $300 \mathrm{~K}$. The temperature was monitored by a silicon diode connected to the temperature control system, which kept the temperature constant to within $0.5 \mathrm{~K}$. 
When the desired temperature was achieved, gas molecules moved from the pre-chamber through a needle valve and filled the chamber. The molecules then froze on the gold electrode of the QCMB when they collided with it from all directions (background deposition). During deposition at a constant rate, because of the constant pressure in the pre-chamber, two interferograms of two polarized (perpendicular to the plane of incidence) He-Ne laser beams at different incidence angles [5] were obtained by two photodiodes. The interference pattern of each laser beam was caused by the interference of the reflections on the top of the $\mathrm{CO}_{2}$ ice and gold surface of the QCMB.

The ice thickness (order of magnitude around $1 \mu \mathrm{m}$ ) and its refractive index were calculated using these two interference patterns. The deposited mass was obtained from the frequency variation of the QCMB, whose area was known. We were then able to calculate the density. Figure 2 shows experimental data that gave the interference curves for each laser beam. The constant slope of the frequency variation of the QCMB indicates a constant rate of deposition.

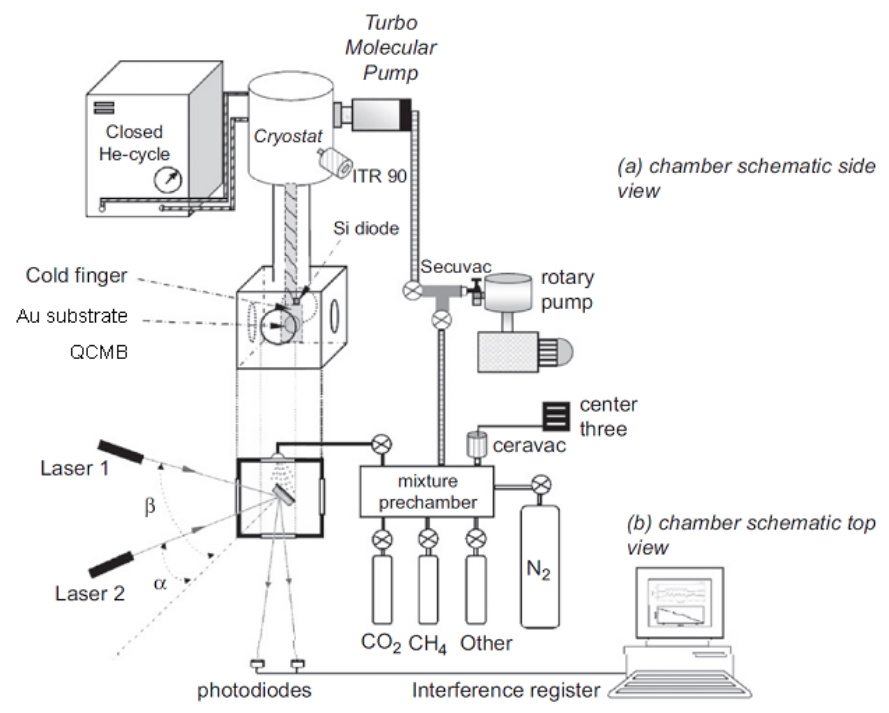

Fig. 1 Diagram of the experimental apparatus

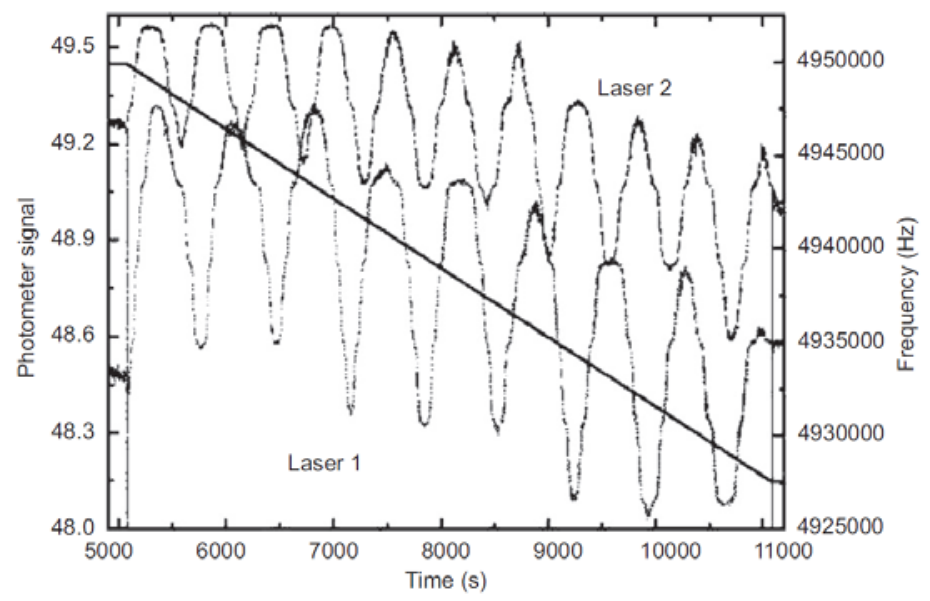

Fig. 2. Data from a deposition experiment [17]: the interference curves of each laser beam from the photometer signals, and the frequency of the QCMB (the solid black line with constant slope) 


\section{RESULTS AND DISCUSSION}

We performed gas deposition experiments in the temperature range of $10-86 \mathrm{~K}$ to form ice of $\mathrm{CO}_{2}$ and determine its density and the real part of the refractive index at $632.8 \mathrm{~nm}$. In Fig. 3, the results [17] indicate that these parameters depend on the deposition temperature.

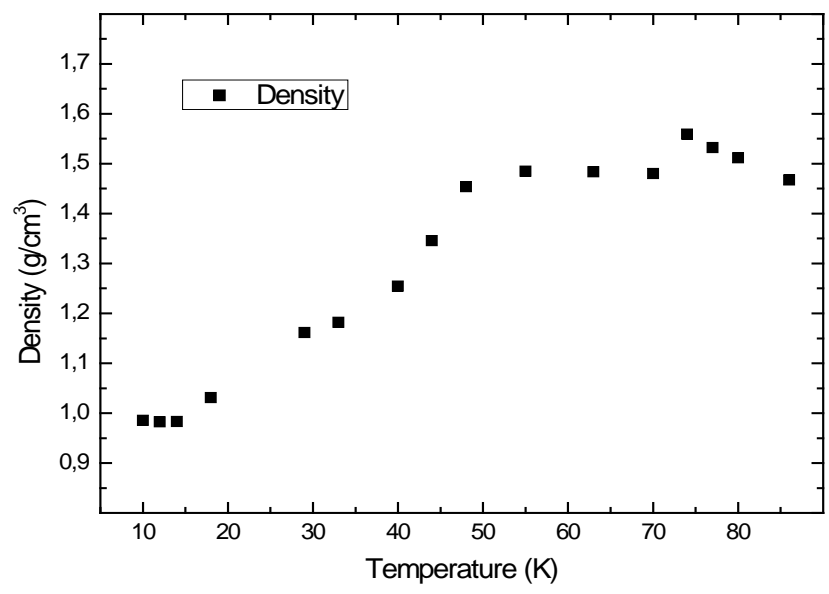

(a)

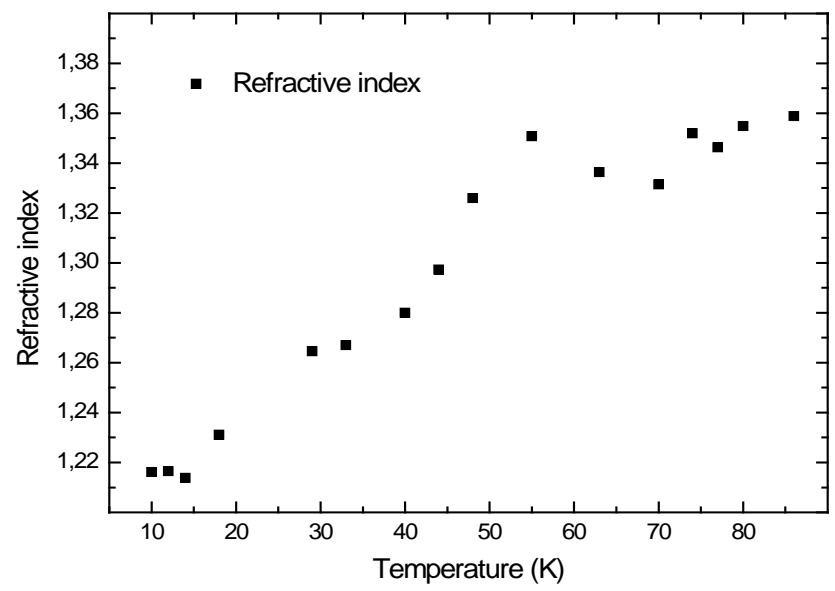

(b)

Fig. 3 Plots [17] of (a) density and (b) refractive index against deposition temperature

From these results, we calculated the polarizability using the Lorentz-Lorenz equation. To obtain the results in volume units, we used the polarizability volume $\alpha$, which is related to the polarizability $\alpha$ by 


$$
\alpha^{\prime}=\frac{\alpha}{4 \pi \varepsilon_{0}}
$$

where $\varepsilon_{0}$ is the permittivity of free space. The Lorentz-Lorenz equation then becomes

$$
\frac{n^{2}-1}{n^{2}+2}=\frac{4 \pi}{3} \frac{N_{A} \alpha^{\prime} \rho}{M}
$$

where $n$ is the refractive index, $N_{\mathrm{A}}$ is Avogadro's number, $\rho$ is density $\left(\mathrm{kg} / \mathrm{m}^{3}\right), M$ is the molar mass $(\mathrm{kg} / \mathrm{mol})$, and $\alpha^{\prime}$ is the polarizability volume $\left(\mathrm{m}^{3}\right)$. Finally, the result of $\alpha^{\prime}$ in converted to $\AA^{3}\left(1 \AA^{3}=10^{-30} \mathrm{~m}^{3}\right)$.

In the derivation of this equation, two mains assumptions have been made. The first is that the electrical field experienced by a molecule is $\mathbf{E}+\mathbf{P} / 3$, where $\mathbf{E}$ is the macroscopic electrical field and $\mathbf{P}$ is the dipolar moment per volume unit. The second is that $n \approx \sqrt{\varepsilon_{r}}$, where $\varepsilon_{r}$ is the real part of the dielectric permittivity of the ice, because for $\mathrm{CO}_{2}$ at the wavelength of the He-Ne laser (632.8 nm) the imaginary part of the refractive index $\hat{n}=n+i k$ is $k \approx 10^{-6}$ [18]. This is because, from the Maxwell equations [19], the dielectric permittivity can be defined as $\widehat{\varepsilon}=\varepsilon_{r}+i\left(\sigma / \varepsilon_{0} \omega\right)=\varepsilon_{r}+i \varepsilon_{i}$ ( $\sigma$ : conductivity) and its relationship with the refractive index is $\hat{n}^{2}=\hat{\varepsilon}$. Using algebraic operations, $\varepsilon_{r}=n^{2}-k^{2}$ and $\varepsilon_{i}=2 n k$.

Figure 4 shows the results for the polarizability volume (hereinafter referred to as polarizability) that we obtained for the different deposition temperatures. From Fig. 4, this parameter remained constant with respect to deposition temperature, so it is not temperature dependent over the range studied. We obtained a value of $\alpha^{\prime}=2.46 \pm 0.06 \AA^{3}$ for the polarizability. We assume that this is the average value of the three diagonal values of the polarizability tensor, i.e., the mean value of the polarizability.

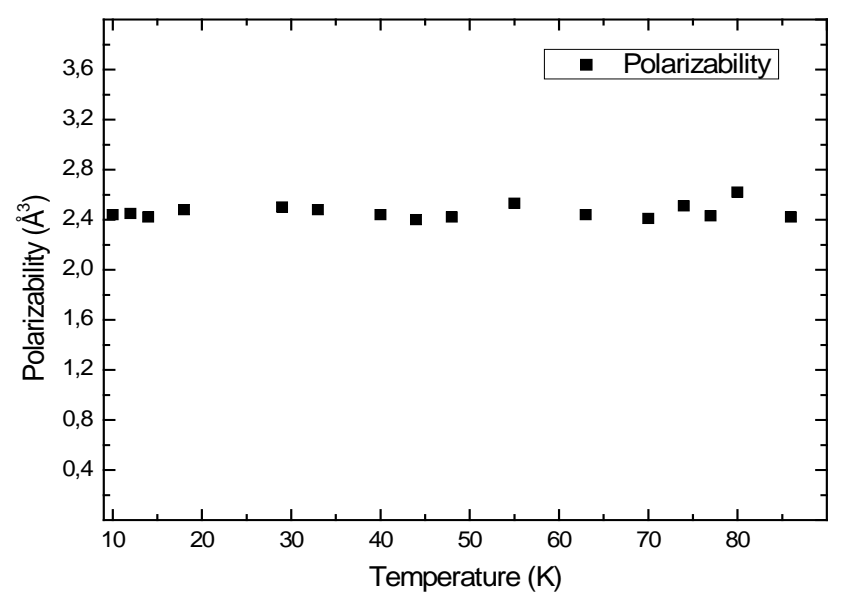

Fig. 4 Relationship between polarizability and deposition temperature 
In Table 1, we compare our results with other published results. All of the previous experiments were performed in the gas phase at different temperature and pressure conditions to our experiments, with either a static electric field or a dynamic from of an electromagnetic wave. Simulation results are also included. It can be seen that, despite the differences in temperature, our results are in agreement with those obtained experimentally and are within the range of those obtained by simulation.

TABLE 1 Comparison between previous experimental and theoretical results, and the results of our own study

\begin{tabular}{|c|c|c|c|c|}
\hline $\begin{array}{c}\text { Conditions: } \\
\text { Phase, Temperature (K), Pressure (bar) }\end{array}$ & $\begin{array}{l}\text { Polarizability } \\
\text { volume }\left(\AA^{3}\right)\end{array}$ & $\begin{array}{l}\text { Electric field } \\
\text { wavelength (nm) }\end{array}$ & Notes & Origin \\
\hline Gas, 307.2, 1 & 2.635 & 546.1 & & $\begin{array}{l}\text { [20] R. H. Orcutt et al. } \\
\text { [2] P. Phillips et al. }\end{array}$ \\
\hline $\begin{array}{l}\text { Gas, 302.5, } 7 \\
\text { Gas, 322.8, } 7\end{array}$ & $\begin{array}{l}2.917 \pm 0.001 \\
2.914 \pm 0.002\end{array}$ & Static & & [21] T. K. Bose et al. \\
\hline Theoretical & 2.352 & & $\begin{array}{l}\text { Mean value for } \\
\text { different GTF Basis set }\end{array}$ & [7] G. Maroulis et al. \\
\hline Theoretical & $\begin{array}{l}\text { Two range: } \\
1.630-2.223 \\
1.926-2.519\end{array}$ & & $\begin{array}{l}\text { RHF Level } \\
\text { Electron correlation }\end{array}$ & [8] M. Lewis et al. \\
\hline Solid, $10-90$ range, $10^{-10}$ & $2.46 \pm 0.06$ & 632.8 & & This study \\
\hline
\end{tabular}

Figure 5 shows our average value, $2.46 \pm 0.06 \AA^{3}$, along with those of other authors, assigning room temperature to the simulations.

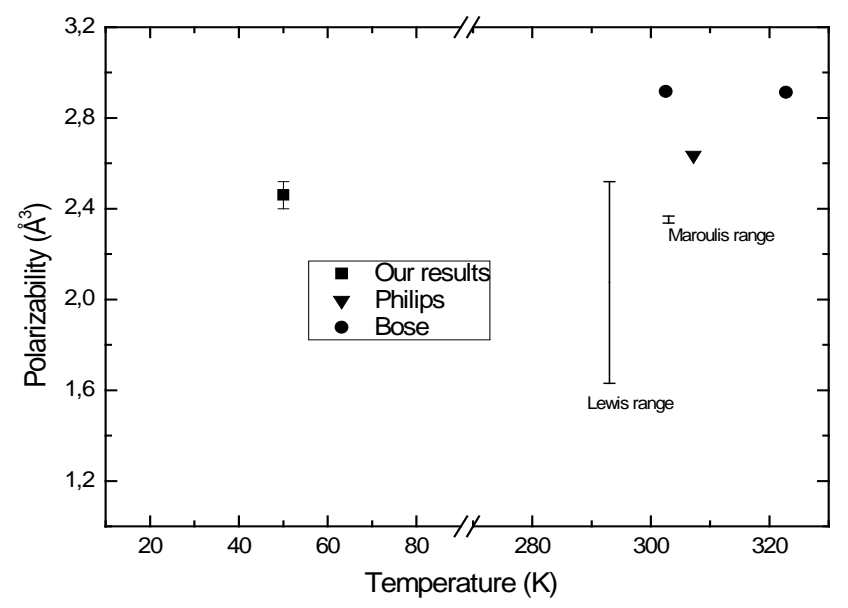

Fig. 5 Plot of polarizability against temperature. For clarity, the results of Maroulis have been displaced $10 \mathrm{~K}$ 
It is necessary to discuss the differences and similarities of the results. Concerning the different states of $\mathrm{CO}_{2}$ in the experiments, there is controversy about the validity of using the Lorentz-Lorenz equation with solids. While some authors believe that it is not applicable [22] or not always applicable to solids [23], others believe that it is valid [24-26]. Because our results agree with those of simulations and experiments in the gas phase, we conclude that the Lorentz-Lorenz equation is applicable for $\mathrm{CO}_{2}$ under the conditions in this study: the solid state, and low temperature and pressure. Concerning the agreement of our result (for $\lambda=632.8$ $\mathrm{nm}$ ) with the static polarizability values, it is necessary to review the different types of polarization. There are three types of polarization: orientational, electronic, and distortional. Because $\mathrm{CO}_{2}$ is a linear molecule and symmetric, it has no permanent dipole moment and does not have orientational polarization. The frequency of the external electrical field from the laser is about $10^{14} \mathrm{~Hz}$

in the visible part of the electromagnetic spectrum. Electronic polarization is caused by distortion of the electronic cloud of the electrons that form the molecule. The frequency of this cloud in the part of the ultraviolet region near the visible region is around $10^{15} \mathrm{~Hz}$ [19]. This frequency is higher than that of the electric field of the laser, and thus the cloud is not affected by changes in the electric field. Therefore, our polarizability value matches the static value.

Distortional polarization arises from distortion of the nuclei by the applied field. The molecule is bent and stretched by the applied field, and the molecular dipole moment changes accordingly. For the typical mass and dimensions of atoms of molecules, the midinfrared region [27] $\left(\sim 10^{13} \mathrm{~Hz}\right)$ encompasses the features of the fundamental molecular vibrational modes. It is too slow to follow the external field, and so for this part of the spectrum the polarizability can be assumed to be zero. Regarding the constant polarizability with changing deposition temperature, it is known that the polarizability is proportional to the size of the molecule, and that it depends on the rovibrational state [28]. Because this state does not change in the temperature range considered, the polarizability also does not change.

\section{CONCLUSIONS}

The validity of our measurements of density and refractive index is supported by the results of previous experiments [25] [29]. Our laboratory has the advantage of being able to simultaneously obtain the density and refractive index under the same conditions. The agreement between our value and the polarizability values obtained by experiments performed for different states and under different conditions of temperature and pressure, and with the simulation results, confirms the validity of the application of the Lorentz-Lorenz equation for $\mathrm{CO}_{2}$ under our experimental conditions. The maximum differences of around $20 \%$ between the different values of polarizability may be caused by inherent errors of the experimental methods.

Furthermore, the polarizability of $\mathrm{CO}_{2}$ remained constant for different temperatures of the formation of the ice. It is interesting to try to understand the relationship between the structure of the solid and the polarizability. There is only one 
reference [30] about the type of structure of solid $\mathrm{CO}_{2}$ at our temperature range, face-centered cubic, but it is at atmospheric pressure. In our temperature range and similar conditions of vacuum, there are two ways to describe the evolution of the structure. By one side, infrared spectroscopy evidences [31] show that when deposition is below $30 \mathrm{~K}$, the structure is amorphous, and when deposition is over $50 \mathrm{~K}$, it is crystalline; once the ice is produced at lower temperatures, the effect of annealing is the production of a more ordered amorphous structure [32] or indeed the crystallization of the solid [31] [33]. The other way to explain the structure is by means the growth of the ice by nucleation [25], with the grains forming little islands of crystal randomly oriented whose size depends directly of temperature; and similarly, the annealing produce the crystallization of the solid, being the source of the energy for this process the increase of temperature or the energy delivered for the molecule gas when impact with the solid. In our laboratory we have observed these structure changes in Thermal Programming Desorption (TPD) experiments [34], where the $\mathrm{CH}_{4}$ from a $\mathrm{CO}_{2}-\mathrm{CH}_{4}$ mixture desorbed at three different temperatures: $35 \mathrm{~K}$ as usual for pure $\mathrm{CH}_{4}, 50 \mathrm{~K}$ when probably the structural change of $\mathrm{CO}_{2}$ occurs, and $90 \mathrm{~K}$ together with the $\mathrm{CO}_{2}$. From our results, we can deduce that polarizability is not affected by this structural change because it is a property associated with the molecule. However, it should also be taken into account that changes in the polarizability tensor cannot be reflected by an experimental value that is assumed to be the mean value of the main diagonal elements of the tensor.

The results presented here have encouraged us to continue this line of work. Future research by our group will center on other molecules in similar conditions and binary mixtures of these molecules.

\section{ACKNOWLEDGMENTS}

This work was supported by the Spanish Ministerio de Economía y Competitividad (FIS2013-48087-C2-2-P).

\section{REFERENCES}

[1] K. J. Miller, J. Am. Chem. Soc., 112, 8543 (1990).

[2] P. Phillips, Proc. R. Soc. Lond. A 97, 225 (1920).

[3] A. C. Newell and R. C. Baird, J. Appl. Phys. 36, 3751 (1965).

[4] D. R. Johnston, G. J. Oudemanns and R. H. Cole, J. Chem. Phys. 33, 1310 (1960); D. R. Johnston and R. H. Cole, J. Chem. Phys. 36, 318 (1962); R. H. Orcutt and R. H. Cole, Physica. 31, 1779 (1965); J. Chem. Phys. 46, 697 (1967).

[5] M. Domingo, C. Millán, M. A. Satorre and J. Cantó, Proceedings of SPIE, Optical Measurements Systems for Industrial Inspection V, Munich, 2007, edited by W. Osten, C. Goreki and E.L. Novak (SPIE, EEUU, 2007), Vol. 6616, p. 6616 4A. 
[6] L. Silberstein, Phil. Mag., 33, 92, 215, 521 (1917); J. Applequist, J. R. Carl and K. K. Fung, J. Am. Chem. Soc. 94, 2952 (1972).

[7] G. Maroulis and A. J. Thakkar, J. Chem. Phys. 93, 4164 (1990).

[8] M. Lewis, Z. Wu and R. Glaser, J. Phys. Chem. A. 104, 11355 (2000).

[9] A. Szabo, N. S. Ostlund, Modern Quantum Chemistry: Introduction to Advanced Electronic Structure Theory (MacMillan Publishing Co., Inc.: New York, 1982); W. L. Hehre, L. Radom, P.v.R. Schleyer and J. A. Pople, Ab Initio Molecular Orbital Theory (John Wiley \& Sons: New York, 1986).

[10] N. J. Bridge and A. D. Buckingham, Proc. R. Soc. Lond. A 295, 334 (1966); G. R. Alms, A. K. Burnham, and W. H. Flygare, J. Chern. Phys., 63, 3321 (1975).

[11] I. R. Gentle, D. R. Laver and G. L. Ritchie, J. Phys. Chem. 93, 3035 (1989); H. A. Stuart and S. v. Schieszl, Ann. Phys. 2, 321 (1948).

[12] A. D. Buckingham and R. L. Dish, Proc. R. Soc. Lond. A 273, 275 (1963).

[13] A. H. England, A. M. Duffin, C. P. Schwartz, J. S. Uejio, D. Prendergast, R. J. Saykally, Chemical Physics Letters, 514, 187 (2011).

[14] T. Takahashi, Encyclopedia of Ocean Sciences, 1, 400 (2001).

[15] R. M. Haberle, B. Mattingly and T. N. Titus, Geophys. Res. Lett. 31, L05702 (2004).

[16] M. Aresta and I. Tommasi, Energy Convers. Mgmt. 38, S373 (1997).

[17] M. A. Satorre, M. Domingo, C. Millán, R. Luna, R. Vilaplana and C. Santonja, Planetary and Space Science 56, 1748 (2008).

[18] S. G. Warren, Appl. Optics, 25, 2650 (1986).

[19] J. R. Reitz, F. J. Mildford and R. W. Christy, Foundations of Electromagnetic Theory (Addison Wesley, 2008) p.434, p506

[20] R. H. Orcutt and R. H. Cole, J. Chem. Phys. 46, 697 (1967).

[21] T. K. Bose and R. H. Cole, J. Chem. Phys. 52, 140 (1970).

[22] P. Lorrain and D. R. Corson, Campos y ondas electromagnéticos (Selecciones Científicas, Madrid, 1972) p.123.

[23] I. Thormählen, J. Straub and U. Grigull, Journal of Physical and Chemical Reference Data, 14, 4, 933 (1985).

[24] M. Born and E. Wolf, Principles of optics (Cambridge University Press, Cambridge, 1999) p.100.

[25] W. Schulze and H. Abe, Chemical Physics 52, 381 (1980).

[26] E. Hecht, Optics (Addison Wesley, San Francisco, 2002) p.66.

[27] W. A. Schutte, Molecules in astrophysics: probes \& processes: abstract book, 1996, Leiden, 1996, edited by Ewine Fleur van Dishoeck (The Netherlands, 1996), IAU symposium 178, p.331.

[28] T. Guella, Thomas M. Miller, J. A. D. Stockdale, B. Bederson, and L. Vušković, J. Chem. Phys. 94, 6857 (1991); T. M. Miller, Handbook of Chemistry and Physics 89th Ed (2009) p.10-193.

[29] K. E. Tempelmeyer and D. W. Mills Jr., J. Appl. Phys. 39, 2968 (1968). 
[30] G. Cardini, P. Procacci, R. Righini, J. Chem. Phys. 117, 355 (1987).

[31] M. Falk, J. Chem. Phys. 86, 560 (1987).

[32] S. A. Sandford and L. J. Allamandola, The Astrophysical Journal, 355, 357 (1990).

[33] R. M. Escribano, G. M. Muñoz Caro, G. A. Cruz-Díaz, Y. Rodríguez-Lezcano et B. Maté , PNAS, 110, 32, 12899 (2013).

[34] R. Luna, C. Millan, M. Domingo and M. A. Satorre, Planetary and Space Science 314, 113 (2008). 